\title{
Understanding the In-Situ Reaction of Cerium Oxide Nanoparticles using Aberration Corrected Exit Wave Restoration and EELS.
}

\author{
S.J.Haigh, ${ }^{* * * *}$ N.P.Young*, H Sawada*** K. Takayanagi, ${ }^{* * * *}$ and A.I.Kirkland* \\ * Department of Materials, University of Oxford, Parks Road, Oxford, OX1 3PH, UK \\ ** Now at: School of Materials, University of Manchester, Grosvenor Street, Manchester, M1 7HS, \\ $\mathrm{UK}$ \\ *** JEOL Ltd.1-2 Mushashino 3-Chome, Akishima, Tokyo 196, Japan. \\ **** Department of Physics, Tokyo Institute of Technology, 2-12-1-H-51 Oh-okayama, Meguro-ku, \\ Tokyo 152-8551, Japan, CREST Japan Science and Technology Corporation, Kawaguchi, Saitama \\ 332-0012 Japan.
}

Cerium dioxide, $\mathrm{CeO}_{2}$ is an important oxide and has a wide range of energy-related applications including automotive three-way catalytic converters (TWCC), as a catalyst and a catalyst support for oxidation and reduction reactions and as an electrolyte for solid oxide fuel cells. The ability of $\mathrm{CeO}_{2}$ to change the oxidation state of $\mathrm{Ce}$ between $\mathrm{Ce}^{3+}$ and $\mathrm{Ce}^{4+}[1]$ combined with the mobility of oxygen anions within the $\mathrm{CeO}_{2}$ (fluorite) lattice at relatively low temperatures [2] has played a critical role in its catalytic functionalities. Cerium dioxide nanoparticles have been shown to have better catalytic performance than bulk material but the detailed mechanism of oxygen buffering is relatively poorly understood. Nanoparticle surfaces are thought to play an important role but prediction of surface configurations in this system using density functional theory, is not straightforward [3]. Atomic force microscopy can provide experimental verification of theoretically predicted structures for bulk single crystals [4] but it has not previously been possible to determine the surface chemistry of active oxide nanoparticles experimentally [5].

In this paper we use spherical aberration corrected transmission electron microscopy (TEM) combined with computational exit wave restoration [6] in order to determine the surface termination of cerium dioxide nanoparticles. We have simulated exit wavefunctions for $\{100\}$ and $\{111\}$ surfaces with different terminations at thicknesses up to $10 \mathrm{~nm}$ using the multislice approach (FIG. 1). By comparison of line scans extracted from these simulations with the experimentally determined phase of the restored exit wavefunction (FIG. 2) we are able to distinguish between oxygen and metal terminated surfaces. Our results verify for the first time theoretical predicted models [5].

It is well known that cerium dioxide is sensitive to the electron beam [1]. Here we take advantage of this electron beam sensitivity to study the change in the surface structure of ceria nanoparticles before and after an in-situ beam induced reduction reaction at ambient temperature. The oxidation state of cerium can be determined using electron energy loss spectroscopy (EELS). We combine our exit wave restoration surface studies with high spatial resolution EELS mapping in order to study changes in the oxidation state of ceria as a function of both electron beam irradiation and distance from the $\{111\}$ surface.

References

[1] L. A. J.Garvie, P. R. Buseck, J. Phys. Chem. Solids 60, (1999) 1943

[2] D. J. M. Bevan, J. Kordis, J. Inorg. Nucl. Chem. 26, (1964) 1509.

[3] A. Migani et. al. Chem. Commu, 46, (2010) 5936 
[4] S. Torbrugge, et. al. Phys. Rev. Lett. 2007, 99, 056101.

[5] A. S. Barnard, A. I. Kirkland. Chem. Mater. 20, (2008) 5460

[6] A.I Kirkland et al. Curr. Appl Phys. 8, (2008) 425.

[7] This work was supported by EPSRC (grant EP/F048009/1), JEOL (UK) Ltd and the Japan Science and Technology Agency (JST) under the CREST project.

(a)

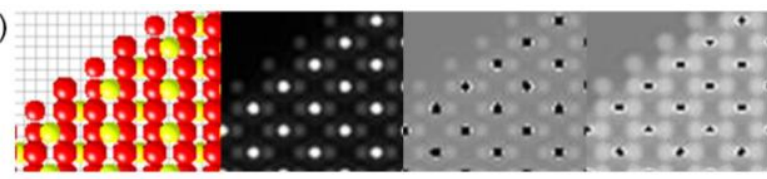

(c)

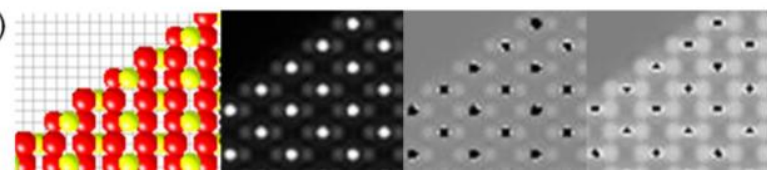

(b)

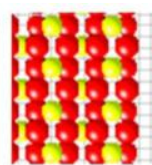

(d)

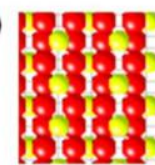

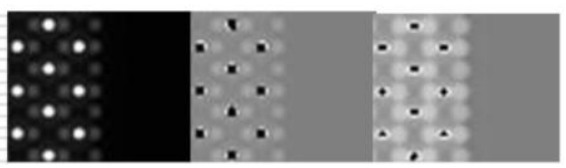

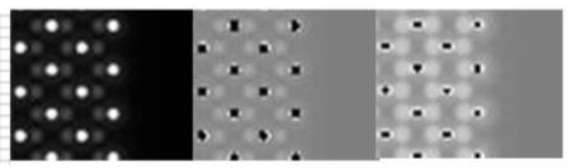

FIG. 1. Simulations of the exit wave for different surface configurations (a) $\{100\}$ oxygen terminated, (b) $\{111\}$ oxygen terminated, (c) $\{100\}$ metal terminated, and (d) $\{111\}$ metal terminated. For each surface the images from left to right represent; the atomic structure with oxygen as dark red atoms and cerium and lighter yellow atoms and the phase of the simulated exit wave with a thickness of $2.7 \mathrm{~nm}, 3 \mathrm{~nm}$, and $5.4 \mathrm{~nm}$ respectively. The viewing direction is [110].

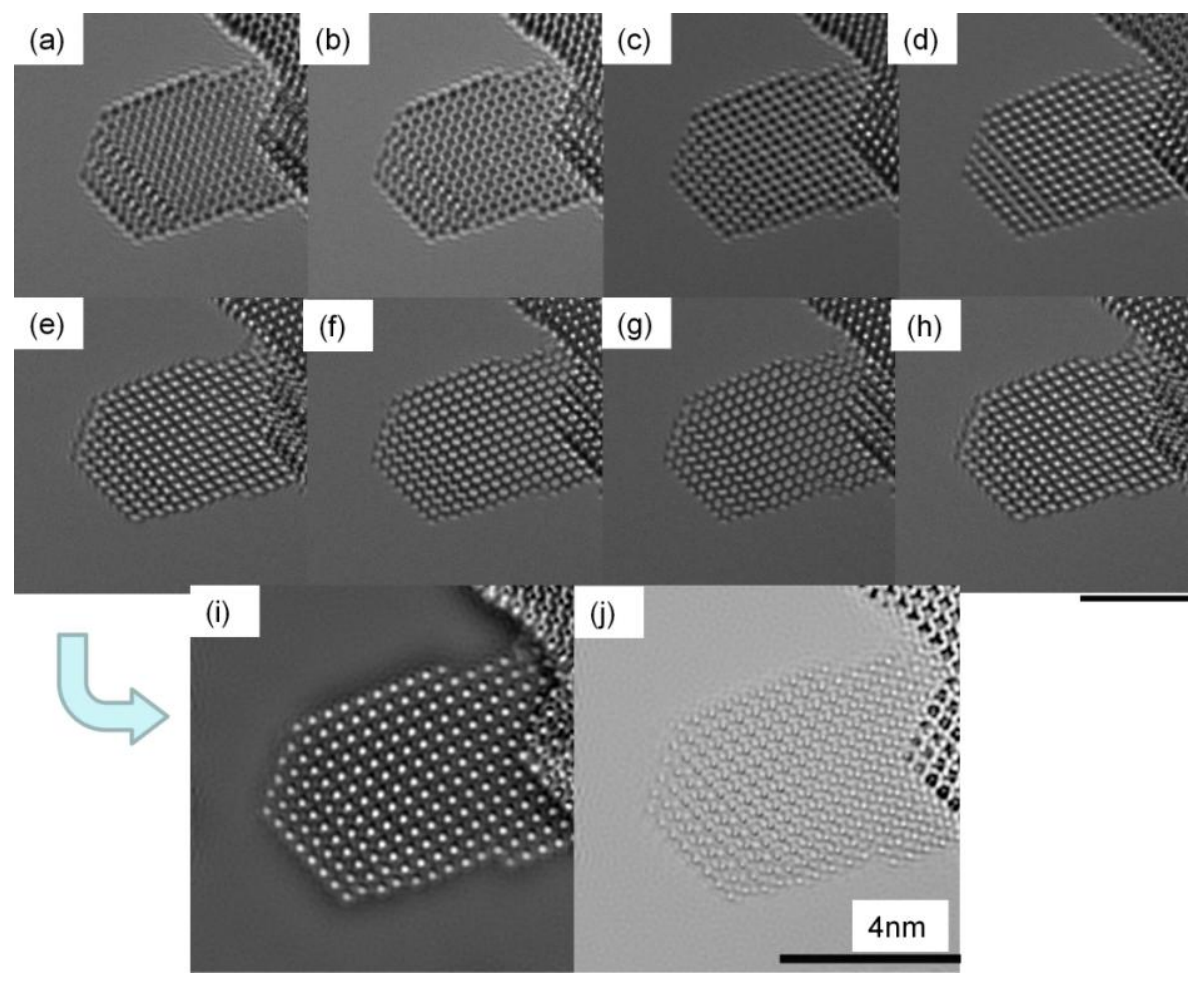

FIG. 2. Selected images from a focal series showing a cerium oxide nanoparticle before electron beam induced reduction. Images were acquired at $200 \mathrm{kV}$ with a spherical aberration corrected to $0.005 \mu \mathrm{m}$. Defocus values for the images were measured as (a)-20.8nm, (b)-18.4nm, (c)-12.6nm, (d)$6.4 \mathrm{~nm},(\mathrm{e})-3.4 \mathrm{~nm}$, (f) $0 \mathrm{~nm},(\mathrm{~g}) 2.5 \mathrm{~nm},(\mathrm{~h}) 16.6 \mathrm{~nm}$. The (i) phase and (j) modulus of the exit wave restored from these images is also shown. 